$11-1-2007$

\title{
Semi Parametric Estimation of Some Reliability Measures of Geometric Distribution
}

\author{
Mathachan Pathiyil \\ Nirmala College \\ E.S. Jeevanand \\ Union Christian College
}

Follow this and additional works at: http://digitalcommons.wayne.edu/jmasm

Part of the Applied Statistics Commons, Social and Behavioral Sciences Commons, and the Statistical Theory Commons

\section{Recommended Citation}

Pathiyil, Mathachan and Jeevanand, E.S. (2007) "Semi Parametric Estimation of Some Reliability Measures of Geometric Distribution," Journal of Modern Applied Statistical Methods: Vol. 6 : Iss. 2 , Article 14.

DOI: $10.22237 /$ jmasm/1193890380

Available at: http://digitalcommons.wayne.edu/jmasm/vol6/iss2/14 


\section{Semi Parametric Estimation of Some Reliability Measures of Geometric Distribution}

\section{Cover Page Footnote}

The first author is thankful to the University Grants Commision, New Delhi India, for the financial support. 


\section{Semi Parametric Estimation Of Some Reliability Measures Of Geometric Distribution}

\author{
Mathachan Pathiyil \\ Nirmala College
}

\author{
E. S. Jeevanand \\ Union Christian College
}

Semi parametric estimators of the survival function, the hazard function, and the mean residual life function of geometric distribution using uncensored and Type II censored samples are obtained. The accuracy of the estimators so obtained is investigated empirically using simulated samples. The results are applied to a real life data set for illustration.

Key words: geometric distribution, hazard function, Kaplan-Meier estimator, least square estimation, mean residual life function, survival function, Type II censoring.

\section{Introduction}

During the past twenty years, manufacturing industries have gone through a revolution in the use of statistical methods to improve product quality. Due to global competition, the industry faces immense pressure for shorter productcycle times, stringent cost constraints, and higher customer expectations for quality and reliability. A natural extension of the revolution in product quality is to focus on product reliability, which is defined as quality over time. Reliability can be defined as the probability that a unit will perform its intended function until a specified point in time under encountered use conditions. The environment in which a product operates is a critical factor in evaluating the

E. S. Jeevanand received the $\mathrm{Ph}$. D. from the Cochin University of Science and Technology, Cochin, Kerala, India. He is Reader in Statistics. His research interests include Bayes estimation, Inference, Reliability and Computational Statistics. He is Associate Editor of the Half Yearly Discourses. Mathachan Pathiyil received his M. Phil from the University of Kerala, Kerala, India. He is Lecturer, Selection Grade, in the department of Statistics, Muvattupuzhaa, Kerala. His research interests include Bayes estimation, Inference and Reliability Analysis. reliability of a product. The design for reliability requires careful consideration of product (process) failure modes. Broadly, failure modes can be classified as those that are anticipated and those that are unanticipated. Generally, engineers focus only on the anticipated failure modes. The main focus, however, of the statistician is in the unanticipated failures and it plays a crucial role in product reliability.

Reliability analysis of devices through failure time data when time is treated as discrete is a recently emerging area of research. Kemp (2004) provided a good discussion on the importance and applications of discrete life distributions. The sophisticated equipment used in the manufacturing process requires accurate measuring devices to record their failures in continuous time. In situations where such measuring instruments are very costly or their availability cannot be ensured, it may be desirable to go in for failure times that are in completed units of time (Xekalaki (1983)). The latter procedure is more desirable, provided the loss of accuracy in replacements of continuous measurements with discrete ones is more than compensated by the gain in terms of other considerations such as money, ease of analysis and time saved etc. Discrete distributions naturally arise when records are taken in completed units of time. The fact that many of the discrete distributions can be closely approximated by continuous distributions adds 
to the utility of the former as models of life length. Also, there is a well developed methodology to separately find the distribution of the integer parts and fractional parts of continuous random variables. This methodology often permits inference on parameters based on count data to be translated to those based on continuous measurements with a reasonable estimate of the margin of error on account of the translation. The geometric distribution owing to its lack of memory property is widely used to model such systems.

Reliability measures of the geometric distribution

An important property of a product or system is its ability to fulfill the intended purpose without failure for a specified period of time under stated conditions. Reliability is a yardstick of the capability of a component to operate without failure when put into service. The survival function, hazard function and mean residual life function are three important notions used extensively for characterizing life distributions.

Let $\mathrm{X}$ denote a discrete random variable in the support of $I^{+}=\{0,1, \ldots\}$ denoting the time to failure of a component. Defining

$$
S(x)=P(X \geq x)
$$

the survival function of $X$ and $f(x)$, the probability mass function of $X$, the hazard rate of $X$ is defined as

$$
\begin{gathered}
h(x)=P(X=x \mid X \geq x) \\
=\frac{f(x)}{S(x)} .
\end{gathered}
$$

and the mean residual life is defined as

$$
\begin{aligned}
r(x) & =E(X-x \mid X>x) \\
& =\frac{1}{S(x+1)} \sum_{y=x+1}^{\infty} S(y) .
\end{aligned}
$$

Suppose that the life span $X$ of the component under observation follows a geometric distribution with probability mass function

$$
f(x)=\theta(1-\theta)^{x}, 0<\theta<1, x=0,1, \ldots
$$

Then

$$
\begin{aligned}
& S(x)=(1-\theta)^{x}, 0<\theta<1, \\
& h(x)=\theta, 0<\theta<1
\end{aligned}
$$

and

$$
r(x)=\frac{1}{\theta}, 0<\theta<1 .
$$

Estimation of the geometric parameter and the reliability measures using uncensored data

$\mathrm{Wu}$ (2001) and Faucher and Tyson (1988) proposed semi parametric estimation of the parameters of exponential and Pareto distributions using the empirical distribution function based on complete samples. The results are further extended, to the study of geometric distribution, by Mathachan and Jeevanand (2005). From (2.5)

$$
\ln \left(S\left(x_{\{i\}}\right)\right)=x_{(i)} \ln (1-\theta) .
$$

Equation (3.1) can be written in the form, $Y_{i}=A X_{i}$, where $A=\ln (1-\theta), X_{i}=x_{(i)}$ and $Y_{(i)}=\ln S\left(x_{(i)}\right)$. By least square procedure, the estimator of $A$ is

$$
\hat{A}=\frac{\sum_{i=1}^{n} \ln \left(S\left(x_{(i)}\right)\right)}{\sum_{i=1}^{n} x_{(i)}}
$$

An estimate of the survival function $S\left(x_{(i)}\right)$ is $\left[1-\hat{F}\left(x_{i: n} ; q\right)\right]$ where $x_{i: n}$ is the $i^{\text {th }}$ order statistic and $\hat{F}\left(x_{i: n}: q\right)=\frac{i}{n}$, the empirical distribution function. In order to avoid $\log (0)$, 
D'Agostino and Stephans (1986) suggested that, $\hat{F}\left(x_{i: n}: q\right)$ can be approximated by $\frac{i-c}{n-2 c+1}$, $i=1,2, \ldots, n$ where $0 \leq c<1$, generally. In this article three popular values for $\mathrm{c}$ are taken and considered in $\mathrm{Wu}$ (2001), Faucher and Tyson (1988), viz. $c=0,0.3$ and 0.5 . Then (3.2) becomes

$$
\hat{A}_{F S}=\frac{\sum_{i=1}^{n} \ln \left(\frac{n+1-c-i}{n+1-2 c}\right)}{\sum_{i=1}^{n} x_{(i)}}
$$

The estimated asymptotic variance of $\hat{A}$ is

$$
\hat{V}\left(\hat{A}_{F S}\right)=\frac{\left(S_{y y}-\frac{S_{x y}}{S_{x x}}\right)}{n-2}
$$

where

$$
\begin{gathered}
S_{x x}=\sum_{i=1}^{n} X_{i}^{2}-n \bar{X}^{2}, S_{y y}=\sum_{i=1}^{n} Y_{i}^{2}-n \bar{Y}^{2} \text { and } \\
S_{x y}=\sum_{i=1}^{n} X_{i} Y_{i}-n \bar{X} \bar{Y} .
\end{gathered}
$$

Estimation of the parameter of the geometric model

An estimator of the parameter of the model (2.4) using the uncensored sample is

$$
\hat{\theta}_{F S}=1-e^{\hat{A}_{F S}}
$$

with estimated asymptotic variance

$$
\hat{V}\left(\hat{\theta}_{F S}\right)=e^{2 \hat{A}_{F S}} \hat{V}\left(\hat{A}_{F S}\right) .
$$

Estimation of the survival function

The corresponding estimator of the survival function is

$$
\hat{S}_{F S}(x)=\operatorname{Exp}\left(x \hat{A}_{F S}\right)
$$

and the estimated asymptotic variance is

$$
\hat{V}_{F S}\left(\hat{S}_{F S}(x)\right)=\hat{A}_{F S} e^{2 x \hat{A}_{F S}} \hat{V}\left(\hat{A}_{F S}\right) .
$$

Estimation of the hazard function

The estimator of the hazard function of the model (2.4) is

$$
\hat{h}_{F S}(x)=\hat{\theta}_{F S}
$$

with estimated asymptotic variance

$$
\hat{V}\left(\hat{h}_{F S}(x)\right)=e^{2 \hat{A}_{F S}} \hat{V}\left(\hat{A}_{F S}\right) \text {. }
$$

Estimation of the mean residual life function

The estimator of the mean residual life function of the model (2.4) is

$$
\hat{r}_{F S}(x)=\left(1-e^{\hat{A}_{F S}}\right)^{-1}
$$

with estimated asymptotic variance

$$
\hat{V}\left(\hat{r}_{F S}\right)=\frac{e^{2 \hat{A}_{F S}}}{\left(1-e^{\hat{A}_{F S}}\right)^{2}} \hat{V}\left(\hat{A}_{F S}\right)
$$

Estimation of the geometric parameter and reliability measures using Type II censored data

With the high reliability products that are common today, testing under normal conditions is time consuming and even expensive. Thus, in life testing experiments, it is a common practice to cease testing before all the components under observation have failed. The resulting sample is a censored sample. Censored data occur frequently in medical research, and estimation of the reliability measures viz. survival function, hazard function and mean residual life function has been an attractive topic when the data are censored. Estimation of hazard function and mean residual life function has drawn less attention than that of the survival function. For the survival function, the KaplanMeier estimator (1958) is a widely used nonparametric estimator. It is strongly consistent and is asymptotically normal (see, Kim, et al., 2005, and Jan, et al., 2005). The focus of this our discussion is estimation of the survival function, hazard function and mean residual life function 
of geometric distribution under Type II censoring.

Estimation of the parameter of the geometric model

A least square estimator is proposed for the parameter of the geometric distribution with survival function (2.5) when the data is censored at a pre defined time T. Suppose $\mathrm{n}$ components with geometric life times are put on test and observed the number of components failed at each time point $t, t+k, t+2 k, \ldots$ up to the time $\mathrm{T}$. Define, $n_{j}$ as the number of components still functioning at the time $t_{j}=t+j k, j=0,1, \ldots, t_{j} \leq T$ and $d_{j} \quad$ as the number of components whose failures occur in the time interval $\left(t_{j-1}, t_{j}\right)$. Then, the KaplanMeier estimator of the survival function $S(t)$ (see Jan et al. (2005)) for a given $t$ is

$$
S *(t)=\prod_{j: t_{(j)} \leq t}\left(\frac{n_{j}-d_{j}}{n_{j}}\right) .
$$

For $t<t_{(1)}, \quad S *(t)=1$. From the survival function (2.5), the following may be written:

$$
\ln \left(S^{*}(t)\right)=t \ln (1-\theta)
$$

Now, (4.2) is of the form $y=A t$, with $y=\ln \left(S^{*}(t)\right)$ and $A=\ln (1-\theta)$. By least square procedures, there is

$$
\hat{A}_{C S}=\frac{\sum_{j=1}^{n} \ln \left(S *\left(t_{j}\right)\right)}{\sum_{j=1}^{n} t_{j}}
$$

and the estimated asymptotic variance of $\hat{A}$ is

$$
\hat{V}\left(\hat{A}_{C S}\right)=\frac{\left(S_{y y}-\frac{S_{x y}}{S_{x x}}\right)}{n-2} .
$$

Consequently, a least square estimator of the parameter $\theta$ is

$$
\hat{\theta}_{C S}=1-e^{\hat{A}_{C S}}
$$

with estimated asymptotic variance

$$
\hat{V}\left(\theta_{C S}\right)=e^{2 \hat{A}_{C S}} \hat{V}\left(\hat{A}_{C S}\right) .
$$

Estimation of the survival function

Substituting (4.3) in (2.5), a least square estimator of the survival function of the geometric distribution considered in (2.4) under Type II censoring scheme is obtained as

$$
\hat{S}_{C S}(x)=e^{x \hat{A}_{C S}}
$$

with asymptotic variance

$$
\hat{V}\left(\hat{S}_{C S}(x)\right)=\hat{A}_{C S} e^{2 x \hat{A}_{C S}} \hat{V}\left(\hat{A}_{C S}\right) .
$$

Estimation of the hazard function

Substituting (4.3) in (2.6), an estimator of the hazard function under the Type II censoring scheme is obtained as

$$
\hat{h}_{C S}=\hat{\theta}_{C S}
$$

and the asymptotic variance of the estimator is

$$
\hat{V}\left(\hat{h}_{C S}\right)=e^{2 \hat{A}_{C S}} \hat{V}\left(\hat{A}_{C S}\right) .
$$

Estimation of the mean residual life function

Substituting (4.3) in (2.7), an estimator of the mean residual life function under this scheme is obtained as

$$
\hat{r}_{C S}=\frac{1}{\hat{\theta}_{C S}}
$$

with asymptotic variance

$$
\hat{\mathrm{V}}\left(\hat{\mathrm{r}}_{\mathrm{CS}}(\mathrm{x})\right)=\frac{e^{2 \hat{A}_{C S}}}{\left(1-e^{\hat{A}_{C S}}\right)^{2}} \hat{V}\left(\hat{A}_{C S}\right) .
$$


Simulation Study

These procedures are assessed by a numerical study based on simulated samples with different values of the parameters of the model. Performances of the proposed estimators are examined empirically by generating samples from the geometric distribution. In the study, the bias of the estimator is defined as

\section{Bias $=$ Average value of the estimate - Parameter value}

and the mean square error (MSE) of an estimator is determined as

$$
\mathrm{MSE}=\text { Variance of the estimator }+(\text { Bias })^{2}
$$

The simulated absolute bias, SD and MSE of the estimators proposed for the reliability measures using uncensored samples of sizes 20, 50 and 100 for 1000 replications corresponding to different choices of the parameter values are given in Tables $1-3$, respectively.

The simulated absolute bias, $S D$ and $M S E$ of the estimators proposed for the reliability measures using Type II censored samples of sizes 20,50 and 100 with different censoring schemes (i. e., for different choices of the censoring time ) for 1,000 replications are given in following tables, Table $4-6$, respectively. These values are computed for different values of the parameters.

A few features observed from Table $1-6$ :

1. For smaller values of $\theta$, the estimators under the two methods proposed have lesser bias and mean square error and a reverse trend is seen for larger values of $\theta$.

2. It seems that the bias and mean square error of all the proposed estimators become smaller as the sample size (or censoring times) increases for a given $\theta$.

Example for assessing the estimators with real data

The pattern of natal dispersal in vertebrate animals is an important factor affecting the genetic and demographic processes within and between populations. The geometric probability distribution is a common way to model the frequency distribution of vertebrate dispersal distances (Porter and Dooley (1993), Greenwood et al., (1979)). They define $X$ as the number of units (home ranges, habitat, nest sites, territories etc. with a fixed diameter) moved before stopping (settling and / or dying) and $\theta$, the probability of stopping while crossing any one unit of habitat before moving to an additional home- range diameter.

For an illustration of the present study, used is the data about the dispersal distance (in units of 200 meters diameter) from natal site to first year breeding site for different categories of 117 one-year-old male great tits given in page 141, Appendix I, Greenwood et al. (1979). The estimators of the geometric parameter and the reliability measures based on the censored and uncensored samples are given in Table 7.

The estimators are computed using Type II censored samples with different choices of the censoring time. The classical estimator of the geometric parameter under the maximum likelihood method of estimation ( $M L E$ ) is

$$
\hat{\theta}_{M L E}=\frac{n}{n+\sum_{i=1}^{n} x_{i}} .
$$

For the above data, the value 0.2566 is obtained. Table 7 suggests that the new semi-parametric estimates suggested are close to the $M L E$ in most cases.

\section{Acknowledgements}

The first author is thankful to the University Grants Commission, New Delhi, India, for the financial support. 
Table 1: Estimators of $S(x)$ using uncensored samples when $x=5$

\begin{tabular}{|c|c|c|c|c|c|c|}
\hline$\theta$ & $S(x)$ & $n$ & $c$ & Bias & $S D$ & $M S E$ \\
\hline \multirow{10}{*}{0.1} & \multirow{10}{*}{0.59049} & \multirow{3}{*}{20} & 0 & 0.0085 & 0.0764 & 0.0059 \\
\hline & & & 0.3 & 0.0182 & 0.0774 & 0.0063 \\
\hline & & & 0.5 & 0.0266 & 0.0782 & 0.0068 \\
\hline & & \multirow{4}{*}{50} & 0 & 0.0141 & 0.0463 & 0.0023 \\
\hline & & & 0.3 & 0.0179 & 0.0466 & 0.0025 \\
\hline & & & 0.5 & 0.0224 & 0.0469 & 0.0027 \\
\hline & & & 0 & 0.0127 & 0.0336 & 0.0013 \\
\hline & & \multirow[t]{3}{*}{100} & 0.3 & 0.0150 & 0.0337 & 0.0014 \\
\hline & & & 0.5 & 0.0176 & 0.0338 & 0.0015 \\
\hline & & & 0 & 0.0339 & 0.0659 & 0.0055 \\
\hline \multirow{8}{*}{0.3} & \multirow{8}{*}{0.16807} & \multirow[t]{3}{*}{20} & 0.3 & 0.0417 & 0.0639 & 0.0058 \\
\hline & & & 0.5 & 0.0481 & 0.0622 & 0.0062 \\
\hline & & & 0 & 0.0394 & 0.0419 & 0.0033 \\
\hline & & \multirow[t]{3}{*}{50} & 0.3 & 0.0439 & 0.0412 & 0.0036 \\
\hline & & & 0.5 & 0.0474 & 0.0406 & 0.0039 \\
\hline & & & 0 & 0.0443 & 0.0301 & 0.0029 \\
\hline & & \multirow[t]{3}{*}{100} & 0.3 & 0.0470 & 0.0297 & 0.0031 \\
\hline & & & 0.5 & 0.0491 & 0.0295 & 0.0033 \\
\hline \multirow{10}{*}{0.5} & \multirow{10}{*}{0.03125} & & 0 & 0.0171 & 0.0178 & 0.0006 \\
\hline & & \multirow[t]{3}{*}{20} & 0.3 & 0.0186 & 0.0164 & 0.0006 \\
\hline & & & 0.5 & 0.0198 & 0.0152 & 0.0006 \\
\hline & & & 0 & 0.0212 & 0.0086 & 0.0005 \\
\hline & & \multirow[t]{3}{*}{50} & 0.3 & 0.0219 & 0.0081 & 0.0005 \\
\hline & & & 0.5 & 0.0225 & 0.0078 & 0.0006 \\
\hline & & & 0 & 0.0229 & 0.0059 & 0.0006 \\
\hline & & \multirow[t]{3}{*}{100} & 0.3 & 0.0233 & 0.0056 & 0.0006 \\
\hline & & & 0.5 & 0.0236 & 0.0055 & 0.0006 \\
\hline & & & 0 & 0.0020 & 0.0019 & 0.0000 \\
\hline \multirow{8}{*}{0.7} & & \multirow[t]{3}{*}{20} & 0.3 & 0.0021 & 0.0016 & 0.0000 \\
\hline & & & 0.5 & 0.0021 & 0.0015 & 0.0000 \\
\hline & & & 0 & 0.0023 & 0.0004 & 0.0000 \\
\hline & 000243 & \multirow[t]{3}{*}{50} & 0.3 & 0.0023 & 0.0003 & 0.0000 \\
\hline & 0.00245 & & 0.5 & 0.0023 & 0.0003 & 0.0000 \\
\hline & & & 0 & 0.0024 & 0.0001 & 0.0000 \\
\hline & & \multirow[t]{2}{*}{100} & 0.3 & 0.0024 & 0.0001 & 0.0000 \\
\hline & & & 0.5 & 0.0024 & 0.0001 & 0.0000 \\
\hline
\end{tabular}


Table 2: Estimators of $h$ using uncensored samples

\begin{tabular}{|c|c|c|c|c|c|c|}
\hline$\theta$ & $h$ & $n$ & $c$ & Bias & $S D$ & $M S E$ \\
\hline \multirow{10}{*}{0.1} & \multirow{10}{*}{0.1} & \multirow{3}{*}{20} & 0 & 0.0039 & 0.0247 & 0.0006 \\
\hline & & & 0.3 & 0.0070 & 0.0254 & 0.0007 \\
\hline & & & 0.5 & 0.0097 & 0.0260 & 0.0008 \\
\hline & & \multirow{3}{*}{50} & 0 & 0.0048 & 0.0145 & 0.0002 \\
\hline & & & 0.3 & 0.0060 & 0.0147 & 0.0003 \\
\hline & & & 0.5 & 0.0074 & 0.0149 & 0.0003 \\
\hline & & \multirow{4}{*}{100} & 0 & 0.0041 & 0.0105 & 0.0001 \\
\hline & & & 0.3 & 0.0049 & 0.0106 & 0.0001 \\
\hline & & & 0.5 & 0.0057 & 0.0107 & 0.0001 \\
\hline & & & 0 & 0.0458 & 0.0735 & 0.0075 \\
\hline \multirow{8}{*}{0.3} & \multirow{8}{*}{0.3} & \multirow[t]{3}{*}{20} & 0.3 & 0.0543 & 0.0747 & 0.0085 \\
\hline & & & 0.5 & 0.0618 & 0.0757 & 0.0096 \\
\hline & & & 0 & 0.0425 & 0.0456 & 0.0039 \\
\hline & & \multirow[t]{3}{*}{50} & 0.3 & 0.0473 & 0.0460 & 0.0044 \\
\hline & & & 0.5 & 0.0512 & 0.0464 & 0.0048 \\
\hline & & & 0 & 0.0448 & 0.0328 & 0.0031 \\
\hline & & \multirow[t]{3}{*}{100} & 0.3 & 0.0478 & 0.0330 & 0.0034 \\
\hline & & & 0.5 & 0.0501 & 0.0332 & 0.0036 \\
\hline \multirow{10}{*}{0.5} & \multirow{13}{*}{0.5} & & 0 & 0.1315 & 0.1199 & 0.0316 \\
\hline & & \multirow[t]{3}{*}{20} & 0.3 & 0.1422 & 0.1193 & 0.0344 \\
\hline & & & 0.5 & 0.1514 & 0.1187 & 0.0370 \\
\hline & & & 0 & 0.1259 & 0.0716 & 0.0210 \\
\hline & & \multirow[t]{3}{*}{50} & 0.3 & 0.1322 & 0.0716 & 0.0226 \\
\hline & & & 0.5 & 0.1373 & 0.0715 & 0.0240 \\
\hline & & & 0 & 0.1297 & 0.0526 & 0.0196 \\
\hline & & \multirow[t]{3}{*}{100} & 0.3 & 0.1336 & 0.0526 & 0.0206 \\
\hline & & & 0.5 & 0.1367 & 0.0526 & 0.0214 \\
\hline & & & 0 & 0.1849 & 0.0882 & 0.0420 \\
\hline \multirow{8}{*}{0.7} & & \multirow[t]{3}{*}{20} & 0.3 & 0.1915 & 0.0853 & 0.0439 \\
\hline & & & 0.5 & 0.1969 & 0.0827 & 0.0456 \\
\hline & & & 0 & 0.1907 & 0.0607 & 0.0401 \\
\hline & & \multirow[t]{3}{*}{50} & 0.3 & 0.1946 & 0.0594 & 0.0414 \\
\hline & 0.7 & & 0.5 & 0.1977 & 0.0584 & 0.0425 \\
\hline & & & 0 & 0.1956 & 0.0427 & 0.0401 \\
\hline & & \multirow[t]{2}{*}{100} & 0.3 & 0.1980 & 0.0422 & 0.0410 \\
\hline & & & 0.5 & 0.1999 & 0.0417 & 0.0417 \\
\hline
\end{tabular}


Table 3: Estimators of $r$ using uncensored samples

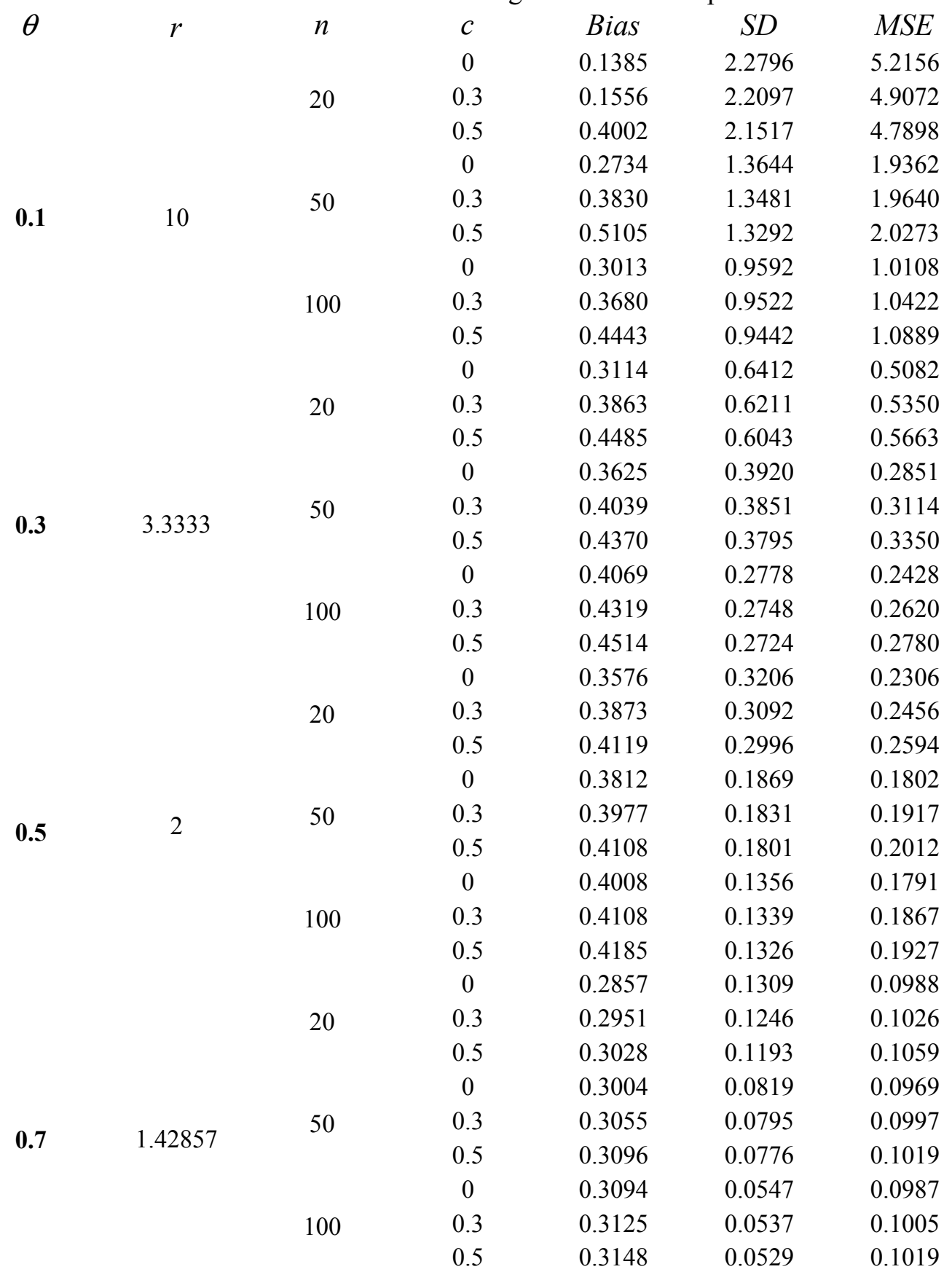


Table 4: Estimators of $S(x)$ using censored samples when $x=5$

\begin{tabular}{|c|c|c|c|c|c|}
\hline$\theta$ & $S(x)$ & $t$ & Bias & $S D$ & $M S E$ \\
\hline \multirow{4}{*}{0.1} & \multirow{4}{*}{0.5905} & 5 & 0.0991 & 0.0118 & 0.0100 \\
\hline & & 10 & 0.0506 & 0.0143 & 0.0028 \\
\hline & & 20 & 0.0240 & 0.0065 & 0.0006 \\
\hline & & 30 & 0.0126 & 0.0108 & 0.0003 \\
\hline \multirow{4}{*}{0.3} & \multirow{4}{*}{0.1681} & 5 & 0.0701 & 0.0059 & 0.0049 \\
\hline & & 10 & 0.0334 & 0.009 & 0.0012 \\
\hline & & 15 & 0.0261 & 0.0066 & 0.0007 \\
\hline & & 5 & 0.0216 & 0.0023 & 0.0005 \\
\hline \multirow{2}{*}{0.5} & \multirow{2}{*}{0.0313} & 7 & 0.0165 & 0.0045 & 0.0003 \\
\hline & & 9 & 0.0114 & 0.0056 & 0.0002 \\
\hline \multirow[t]{2}{*}{0.7} & \multirow[t]{2}{*}{0.0024} & 5 & 0.0019 & 0.0002 & 0.0000 \\
\hline & & 7 & 0.0005 & 0.0004 & 0.0000 \\
\hline
\end{tabular}

Table 5: Estimators of $h$ using censored samples

$\begin{array}{cccccc}\theta & h & \text { Bias } & S D & \text { MSE } \\ & & 5 & 0.0325 & 0.0042 & 0.0011 \\ \mathbf{0 . 1} & 0.1 & 10 & 0.0160 & 0.0047 & 0.0003 \\ & & 20 & 0.0074 & 0.0020 & 0.0001 \\ & & 30 & 0.0039 & 0.0033 & 0.0000 \\ \mathbf{0 . 3} & 5 & 0.0718 & 0.0076 & 0.0052 \\ & & 10 & 0.0305 & 0.0088 & 0.0010 \\ & & 15 & 0.0233 & 0.0062 & 0.0006 \\ \mathbf{0 . 5} & 5 & 0.1060 & 0.0203 & 0.0117 \\ & 0.5 & 7 & 0.0721 & 0.0262 & 0.0059 \\ \mathbf{0 . 7} & & 9 & 0.0454 & 0.0241 & 0.0026 \\ & & 5 & 0.0772 & 0.0169 & 0.0062 \\ & 0.7 & 7 & 0.0132 & 0.0117 & 0.0003\end{array}$


Table 6: Estimators of $r$ using censored samples

\begin{tabular}{|c|c|c|c|c|c|}
\hline \multirow[t]{2}{*}{$\theta$} & $r$ & $t$ & Bias & $S D$ & $M S E$ \\
\hline & \multirow{4}{*}{10} & 5 & 2.4462 & 0.2343 & 6.039 \\
\hline \multirow{3}{*}{0.1} & & 10 & 1.3706 & 0.3536 & 2.0036 \\
\hline & & 20 & 0.6898 & 0.1782 & 0.5076 \\
\hline & & 30 & 0.3674 & 0.3133 & 0.2332 \\
\hline \multirow{4}{*}{0.3} & \multirow{4}{*}{3.3333} & 5 & 0.6425 & 0.0549 & 0.4159 \\
\hline & & 10 & 0.3060 & 0.0826 & 0.1005 \\
\hline & & 15 & 0.2393 & 0.0605 & 0.0609 \\
\hline & & 5 & 0.3485 & 0.054 & 0.1243 \\
\hline \multirow{2}{*}{0.5} & \multirow{2}{*}{2} & 7 & 0.2492 & 0.0804 & 0.0686 \\
\hline & & 9 & 0.1635 & 0.0836 & 0.0337 \\
\hline \multirow[t]{2}{*}{0.7} & \multirow{2}{*}{1.4286} & 5 & 0.1414 & 0.0278 & 0.0208 \\
\hline & & 7 & 0.0261 & 0.0229 & 0.0012 \\
\hline
\end{tabular}

Table 7: Estimators of the parameter and the reliability measures using the real data when $x=5$

$\begin{array}{ccccc}\begin{array}{c}\text { Censoring } \\ \text { time } t\end{array} & \begin{array}{c}\text { Estimate } \\ \text { of } \theta\end{array} & \begin{array}{c}\text { Estimate of } \\ S(x)\end{array} & \begin{array}{c}\text { Estimate } \\ \text { of } h\end{array} & \begin{array}{r}\text { Estimate } \\ \text { of } r\end{array} \\ \mathbf{5} & 0.3345 & 0.1305 & 0.3345 & 2.9892 \\ \mathbf{1 0} & 0.3229 & 0.1424 & 0.3229 & 3.0973 \\ \mathbf{1 5} & 0.3102 & 0.1562 & 0.3102 & 3.2298 \\ \begin{array}{c}\text { Uncensored } \\ \text { data }\end{array} & 0.2912 & 0.1790 & 0.2912 & 3.4347\end{array}$




\section{References}

D’Agostino, R. B. \& Stephans, M. A. (1986). Goodness of Fit Techniques. Marcel Dekker, Inc., New York.

Faucher, B. \& Tyson, W.R. (1988). On the determination of Weibull parameters. Journal of Material Science Letters, 7, 11991203.

Greenwood, P. J. Harvey, P. H. \& Perrins, C. M. (1979). The role of dispersal in the Great Tit (Parus Major): The causes, consequences and heritability of natal dispersal. Journal of Animal Ecology, 48, 123-142.

Jan, B., Shah, S.W.A., Shah, S. \& Qadir, M. F. (2005). Weighted Kaplan - Meier estimation of survival function in heavy censoring. Pakistan Journal of Statistics, 21 (1), 55- 83.

Kaplan, E. L. \& Meier, P. (1958). Nonparametric estimation from incomplete observations. Journal of the American Statistical Association, 53, 457- 481.
Kemp, A.W. (2004). Classes of discrete life distributions. Communications in StatisticsTheory and Methods, 33(12), 3069-3093.

Kim, C., Bae, W., Cho, H. \& Park, B. U. (2005). Nonparametric hazard function estimation using the Kaplan - Meier estimator. Nonparametric Statistics, 17 (8), 937 - 948.

Mathachan Pathiyil \& Jeevanand, E. S. (2005). Estimation of some reliability measures of geometric distribution by least square procedure. Recent Advances in Statistical Theory and Applications, 1, $117-125$.

Porter, J.H \& Dooley J.L (1993). Animal dispersal patterns: A reassessment of simple mathematical models. Ecology, 74(8), 2436-2443.

$\mathrm{Wu}$, J.W. (2001). A note on determining the number of outliers in exponential sample by least square procedure. Statistical papers, 42(4), 489-503.

Xekalaki, E. (1983). Hazard functions and life distributions in discrete time. Communications in Statistics - Theory and Methods, 12, 2503-2509. 